\title{
A Taxonomy of Equilibria in Probabilistic Coalitions
}

\author{
Edward A. Billard and Joseph C. Pasquale \\ Department of Computer Science and Engineering \\ University of California, San Diego \\ La Jolla, CA 92093-0114
}

\begin{abstract}
A model is presented where uncertainty exists concerning whether agents work together in a coalition or work alone, capturing the uncertainty for group behavior in political, economic or distributed computing systems. The uncertainty is modeled as a probability of group formation and the performance of the system is modeled as payoffs in a simple game matrix. Both the level of uncertainty and the game payoffs determine the type of equilibria in the actions of the decision-makers. All equilibria are classified into one of three types where each type creates a different level of difficulty in the agents' decisionmaking.
\end{abstract}

\section{INTRODUCTION}

We present a model where there is uncertainty whether agents work together in a coalition or work alone; we call these probabilistic coalitions. The model captures the uncertainty for group behavior in political, economic or distributed computing systems. Agents, or players, in these systems seek to improve performance by working with other compatible agents; however, the agents are uncertain as to the explicit group formation.

The cause of the uncertainty may be random influences of environmental factors or adaptive strategies of distributed agents. Regardless of the source of the uncertainty, we use the clustering paramelere to model the probability that two agents will work together in a coalition. Otherwise, with probability 1-c, each agents works alone. Our choice of a probabilistic stralegy for group formation is influenced by the randommess of biological interaction [1] and computational ecologies [2].

'The performance of the system is modeled as payoffs it a simple game matrix. 'The magnitudes of the payoffs for working together in a coalition represent a measure of the synergy between the decision-making agents. However, the placement of the payoffs in the ganc models the degree of difficulty to reach this potential. Although

Manuscript reseived July 15, 19993. This work was supported in part by the National Sirence Foundation, IBN, NCR and TRW. Mailing ardoress for lidward Billard is I Iniversity of Aizu. AizuWakamatsu ( ity, lukushima P'refocture 965, Japan. decision-making is easier for agents working alone, the payoffs are only proportional to the performance of working alone.

The probability $c$ of group formation induces an average game and the equilibria can be classified into one of three types, each with different implications for decisionmakers in the context of dynamic group formation. The domain for the value of $c$ creates a landscape of potential performance but the agents must make decisions that achieve equilibrium to realize this potential. Some applications, as represented by games, do not create any obstacles to the best decisions, while other applications at least require that the agents establish a new equilibrium. And last, some applications, at particular values of $c$, do not have pure equilibria available. Appropriate strategies must be available to handle the type of equilibria available in the system. All games can be categorized into one of these three types and our taxonomy provides a simple presentation and summary of potential applications. In Section V, the optimal probability, with respect to equilibrium actions, is determined analytically, suggesting possible search strategies. Our taxonomy has implications for the dynamic formation of groups in distributed systems, especially for those mechanisms that seek equilibrium $[3,4]$.

The paper is organized as follows: Section II presents an example of a distributed political system. Section III describes the model; Section IV illustrates games with various stability of the equilibria; Section $V$ analyzes the optimal clustering parameter; Section VI presents a taxonomy of coalition games based on normalized games. Our conclusions are presented in Section VII.

\section{Example: A Distributed Political System}

For motivational purposes, we present a simplified model of a distributed political system. The payoffs in the game are identical to those used to describe a load balancing problem in a distributed computing system [5]. The key concept is that agents attempt to make the best possible decision in the face of uncertainty regarding group formation.

The Soviet Empire has moved from a centralized system to a distributed system but has suffered due to a 
lack of cooperation. The Los Angeles Times reported on September 3, 1991 that the Soviet Republics could "each choose how to cooperate with the others or not to cooperate at all". Indeed, the Baltic States of Estonia, Latvia and Lithuania readily agreed to form a coalition due to similar political, economic and social goals. However, the Commonwealth of Independent States, with Russia in the lead, seems a very weak coalition due to divergent goals and mutually conflicting constraints. There is a high degree of uncertainty about the actual membership of the coalition with Ukraine being a possible defector. We could say that there is a probability that Ukraine will join and that there is an overall probability of a particular coalition formation. This uncertainty makes other decision-making more difficult (see the problem of food production below).

For now, many Eastern European countries such as Hungary and Romania operate independently, or as singletons. These structures are presented in Fig. 1. If we consider each country as an agent, then we say that all agents are searching for the optimal group formation. The important point is that a large coalition has the potential for synergy at the risk of some poor results due to possibly conflicting collective actions whereas singletons have predictability due to low-risk, or independent, actions.

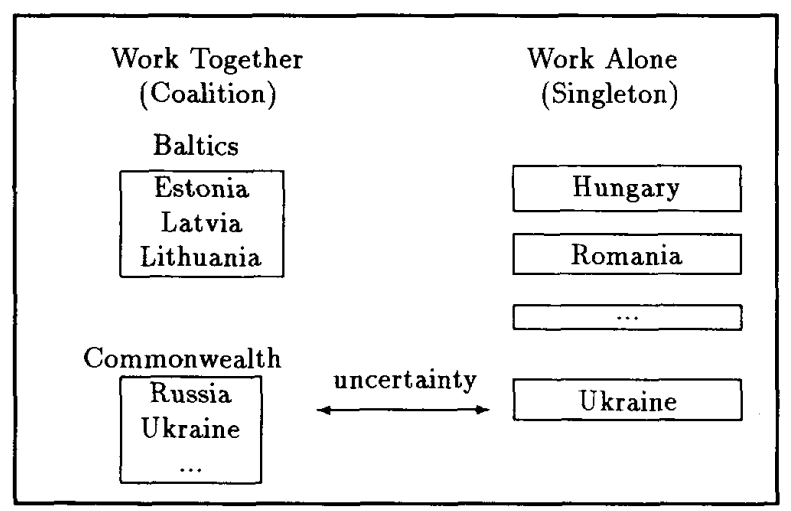

Fig. 1: Soviet Political Coalitions

The decision-making of the agents appears to be adaptive; that is, there is no predefined plan to reach the optimal group formation but rather small steps made in one direction while observing the dynamic environment and the decisions of other agents. There is a lack of communication that creates an uncertainty in the strategies of the various agents.

Let us continue this example but with just two countries and suppose they are concerned with feeding their populations during the winter; the alternatives for calorie production are shown in Fig. 2. Each cointry is rep- resented by a single decision-maker, or agent (perhaps in the form of a committee), who must choose whether to join in a coalition with the other agent or to act as a singleton (if either chooses to act as a singleton, then both must act independently). The two structures of working together or working alone have associated payoffs in matrix or vector form, respectively.

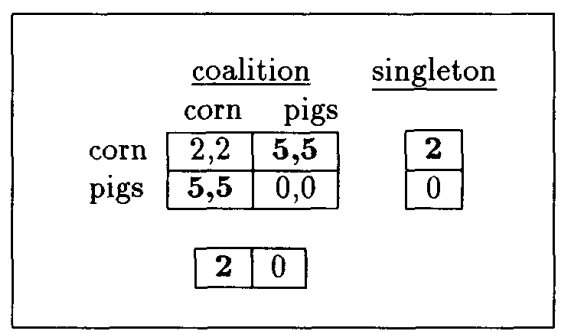

Fig. 2: Average Calories for Population (in $\mathrm{K} /$ day)

Each agent must also decide whether to plant corn or raise pigs, exclusively. We assume many harvesting seasons, or stages in the game, when this decision must be made. Corn can be eaten by the population or by the pigs. Corn has a low caloric value for humans. Pigs have a high caloric value for humans but must eat corn to grow. The objective is to consume the most calories to survive the winter. The best decision is for the agents to form a coalition and for one partner to grow corn and the other to raise pigs. The corn can be fed to the pigs, which can then be shared by both populations. In this case, the agents (hence the population) receive 5000 calories per day (needed for hard labor in a cold climate). If the agents both decide to plant corn as a coalition, then there is no additional benefit to the coalition and each receives 2000 calories per day.

As singletons, the best decision is to plant corn because there is no hope of coordination and corn will provide a subsistence diet. In any case, the worst decision is to raise pigs when there is no corn available as feed. Note that Fig. 2 shows the equilibria in boldface; that is, there is no motivation, in terms of payoff, for a player to alter its action assuming that the other player does not do so. There are equilibria in both the coalition and the singletons; the problem for the decision-makers is the uncertainty in which structure is appropriate. Next, we formalize the concept of a coalition and the uncertainty that exists regarding group formation.

\section{The Model of Probabilistic Coalitions}

We define a coalition as a group whose members have agreed to work together and who expect a payoff, or resultant performance, that is based upon their collective 
decisions. Otherwise, a decision-maker may decide to act alone as a singleton and receive payoffs based only on its own decisions. For a two agent system, these payoffs are modeled as a game with a set of matrices containing the payoffs associated with each combination of actions:

$$
D=\left\{D^{12}, D^{21}, D^{1}, D^{2}\right\}
$$

where

$$
\begin{array}{cc}
D^{12}=\left[\begin{array}{cc}
D_{00}^{12} & D_{01}^{12} \\
D_{10}^{12} & D_{11}^{12}
\end{array}\right], \quad D^{21}=\left[\begin{array}{ll}
D_{00}^{21} & D_{01}^{21} \\
D_{10}^{21} & D_{11}^{12}
\end{array}\right], \\
D^{1}=\left[\begin{array}{l}
D_{0}^{1} \\
D_{1}^{1}
\end{array}\right], & D^{2}=\left[\begin{array}{c}
D_{0}^{2} \\
D_{1}^{2}
\end{array}\right] .
\end{array}
$$

The matrices $D^{12}$ and $D^{21}$ represent the payoffs to player 1 and player 2 , respectively, when members of the coalition. When acting as singletons, $D^{1}$ and $D^{2}$ represent the payoffs to players 1 and 2 , respectively. For example, $D_{\alpha_{1} \alpha_{2}}^{12}$ is the payoff to player 1 when choosing action $\alpha_{1}$ while player 2 chooses action $\alpha_{2}$, where $\alpha_{i} \in\{0,1\}$. As singletons, $D_{\alpha_{2}}^{2}$ is the payoff to player 2 when choosing action $\alpha_{2}$. The structure of a game refers to the size, or dimension, of the game matrix; in a 2-agent system, the structure may either be a 2-player matrix or a 1-player matrix, i.e. a vector. The matrix signifies that a group has action dependency and the vector signifies that agents working alone have action independency. (Note the singleton vector for payoffs to an isolated player is more general than the fixed payoff of 1 in a prisoner's dilemma [6]).

A probabilistic coalition is a coalition in which the formation decision is based upon the result of a random device that accepts as input the clustering parameter $c \in[0,1]$. A probabilistic coalition is similar to an "imperfect coalition" [7] except that the decision to desert is made randomly. An average 2-player game, $\bar{D}=\left\{\overline{D^{12}}, \overline{D^{21}}\right\}$, is induced by the clustering parameter where

$$
\begin{gathered}
\overline{D^{12}}=D^{12} \cdot c+\left[D^{1} D^{1}\right](1-c) \\
\overline{D^{21}}=D^{21} \cdot c+\left[\begin{array}{l}
\left(D^{2}\right)^{T} \\
\left(D^{2}\right)^{T}
\end{array}\right](1-c) .
\end{gathered}
$$

An example of an average game induced by a clustering parameter $(c=.75)$ is shown in Fig. 3 . Note that the singleton matrices, $D^{1}$ and $\left(D^{2}\right)^{T}$, must be replicated to allow for the matrix addition with $D^{12}$ and $D^{21}$, respectively. The induced game has different payoffs and equilibria based on $c$. Obviously, if $c=0$ or $c=1$, then the payoffs are completely determined by the singleton or 2-player matrix, respectively.

\section{Categories of Stability}

The results of running the program in Fig. 4 on several games are shown in Figs. 5 to 8 . The plots show the combined payoff for both players. or gain $G$, at equilibrium

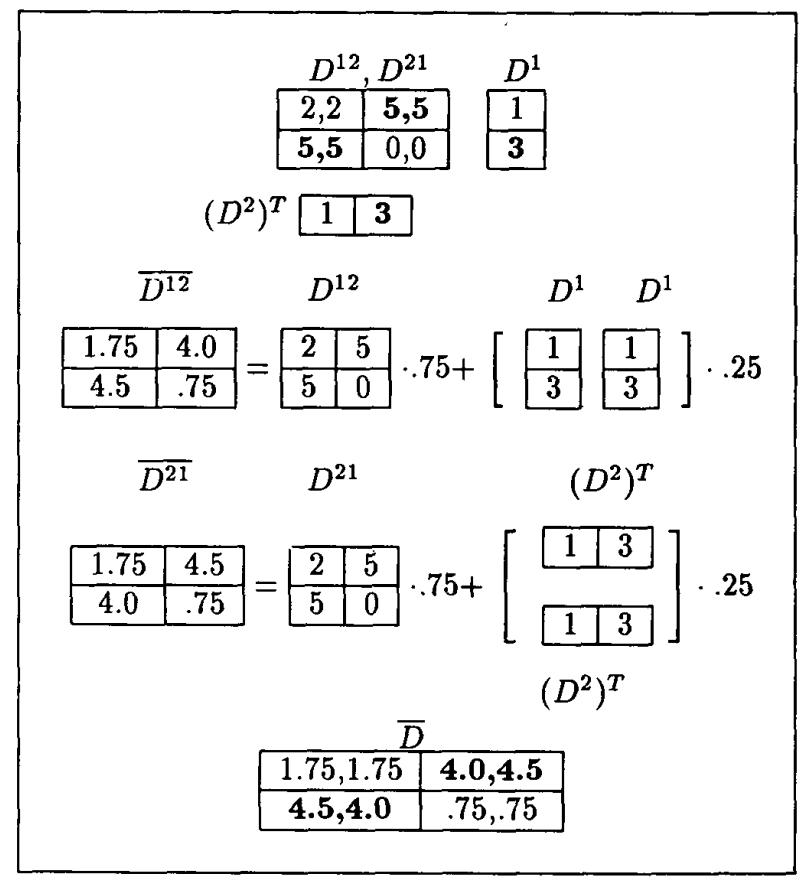

Fig. 3: Average Game Induced by Clustering Parameter $(c=.75)$

over the domain of the clustering parameter $c$. In each figure, one plot shows a game played with the maximum singleton payoff of 1 and another plot shows the same game with a maximum singleton payoff of 4 . The higher singleton payoff games induce equilibria which have a negative slope as a function of the clustering parameter. As the clustering parameter tends to zero, higher payoffs result from the singleton games.

The equilibria plots indicate patterns in the stability of the games. All games, and all equilibria, may be classified into one of three categories: strongly stable, stable, or weakly stable. The stability of a game is determined by how its various equilibria extend over the domain of

$$
\begin{aligned}
& \text { for } \alpha_{1}, \alpha_{2} \in\{0,1\} \\
& \text { for } c \in[0,1] \\
& \quad \bar{D}^{12}=D^{12} \cdot c+\left[D^{1} D^{1}\right](1-c) \\
& \bar{D}^{21}=D^{21} \cdot c+\left[\begin{array}{c}
\left(D^{2}\right)^{T} \\
\left.\left(D^{2}\right)^{T}\right](1-c) \\
\text { if } \bar{D}_{\alpha_{1} \alpha_{2}}^{12} \geq \bar{D}_{1-\alpha_{1}, \alpha_{2}}^{12} \text { and } \bar{D}_{\alpha_{1} \alpha_{2}}^{21} \geq \bar{D}_{\alpha_{1}, 1-\alpha_{2}}^{21} \\
\quad P \operatorname{lot}\left(\bar{D}_{\alpha_{1} \alpha_{2}}^{12}+\bar{D}_{\alpha_{1} \alpha_{2}}^{21}\right) \text { at } c
\end{array}\right.
\end{aligned}
$$

Fig. 4: Pseudocode for Determining Equilibria 
clustering values. Note that all games have at least some domain of stability since an equilibrium always exists in the singleton vectors and the average game reduces to the singleton vectors when $c=0$. Fig. 5 shows strongly stable games where a single action pair is in equilibrium for all values of the clustering parameter. (We would also call this equilibrium strongly stable). The singleton games merely reinforce the dominant strategies of the 2player game. In this case, the clustering parameter does not affect decision-making: the best decisions working together in a coalition are the best decisions working alone.

Fig. 6 shows stable games where there are four equilibria. At any clustering value, there is some action pair that is in equilibrium, but no single equilibrium exists for all values of the clustering parameter. (We also call each of these equilibria stable because they are adjacent to each other.) This suggests that a hill-climbing technique [3] which loses equilibrium due to a change in the clustering parameter must find another hill to climb.

Fig. 7 shows weakly stable games where there are no equilibria for certain values of the clustering parameter. Although all non-zero-sum games have an equilibrium in, at least, mixed strategies [8], we only plot equilibria in pure strategies. The analytic treatment makes no prediction concerning the behavior of players under the condition that a pure strategy equilibrium does not exist. A hill-climbing technique [5] might try all action pairs in vain at certain values of the clustering parameter, a gross approximation of a mixed strategy.

We also call the equilibria that have an edge along the gap weakly stable. In general, any equilibrium that is not adjacent to another (with the exception of a strongly stable equilibrium) is referred to as weakly stable. (Note that all games will at least have a pure strategy equilibrium at $c=0$ since the singleton vectors always provide such an equilibrium.)

Fig. 8 shows games where two equilibria exist at the same clustering parameter. Each of these action pairs represent concurrent equilibria. A hill-climbing technique may be biased towards one particular action pair based upon initialization. The games in the first two plots of Fig. 8 are classified as strongly stable with its strongly stable equilibrium being concurrent to its weakly stable equilibrium. The last two plots show games that are classified as stable with one of its stable equilibria being concurrent to a weakly stable equilibrium. Games with concurrent equilibria are always classified according to the strongest equilibrium present.

\section{Optimality of Asymptotic Coalitions}

Figs. 5 to 8 show straight line plots with positive or negative slopes. This suggests that the optimal gain

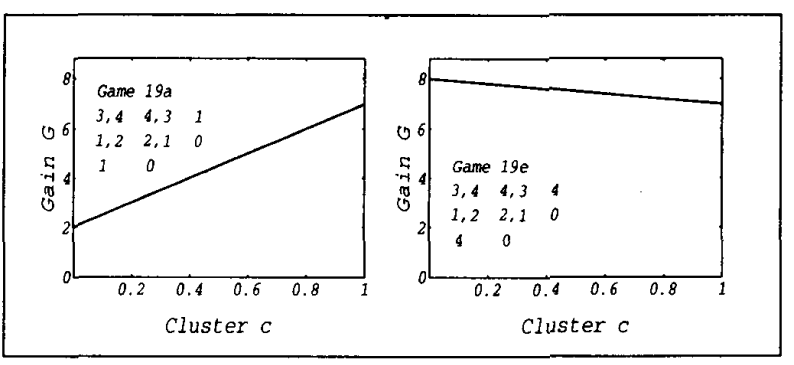

Fig. 5: Games with One Strongly Stable Equilibrium

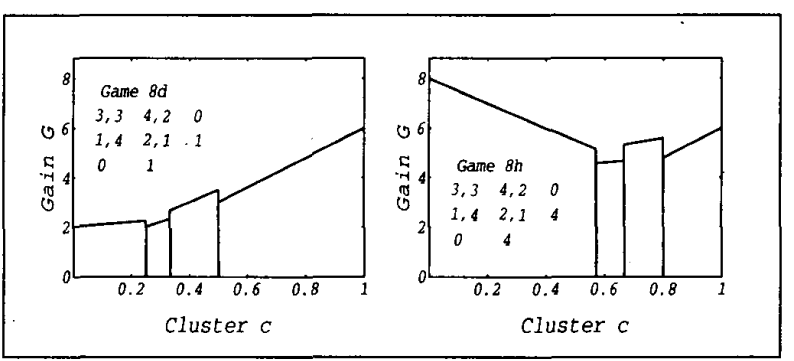

Fig. 6: Games with Four Stable Equilibria

occurs when the clustering parameter $c=1$ or $c=0$ (i.e. asymptotic coalitions are optimal). However, the gaps in the weakly stable equilibria in Fig. 7 show that a pure equilibrium might be lost while searching for the optimum in a repeated game.

Theorem 1 (Asymptotic Coalitions) Given a coalition game, $D=\left\{D^{12}, D^{21}, D^{1}, D^{2}\right\}$ and action pair $\left(\alpha_{1}, \alpha_{2}\right)$, the gain $G\left(\alpha_{1}, \alpha_{2}, c\right)$ in the game, $\bar{D}$, induced by the clustering parameter $c$ is optimized at:

$$
\begin{gathered}
G^{*}=\max _{c} G\left(\alpha_{1}, \alpha_{2}, c\right)= \\
\begin{cases}D_{\alpha_{1} \alpha_{2}}^{12}+D_{\alpha_{1} \alpha_{2}}^{21} & \text { if } c=1 \text { and } \frac{d G\left(\alpha_{1}, \alpha_{2}, c\right)}{d c} \geq 0 \\
D_{\alpha_{1}}^{1}+D_{\alpha_{2}}^{2} & \text { if } c=0 \text { and } \frac{d G\left(\alpha_{1}, \alpha_{2}, c\right)}{d c} \leq 0\end{cases}
\end{gathered}
$$

and the action pair $\left(\alpha_{1}, \alpha_{2}\right)$ will be in equilibrium if, and only if,

$$
\begin{aligned}
& c \geq c^{12}\left(\alpha_{1}, \alpha_{2}\right)=\frac{D_{1-\alpha_{1}}^{1}-D_{\alpha_{1}}^{1}}{D_{\alpha_{1} \alpha_{2}}^{12}-D_{\alpha_{1}}^{1}-D_{1-\alpha_{1}, \alpha_{2}}^{12}+D_{1-\alpha_{1}}^{1}} \\
& c \geq c^{21}\left(\alpha_{1}, \alpha_{2}\right)=\frac{D_{1-\alpha_{2}}^{2}-D_{\alpha_{2}}^{2}}{D_{\alpha_{1} \alpha_{2}}^{21}-D_{\alpha_{2}}^{2}-D_{\alpha_{1}, 1-\alpha_{2}}^{21}+D_{1-\alpha_{2}}^{2}} .
\end{aligned}
$$

Proof: The average game induced by the clustering parameter $c$ is

$$
\bar{D}=\left\{\overline{D^{12}}, \overline{D^{21}}\right\}
$$

where

$$
\overline{D^{12}}=D^{12} \cdot c+\left[D^{1} D^{1}\right](1-c)
$$




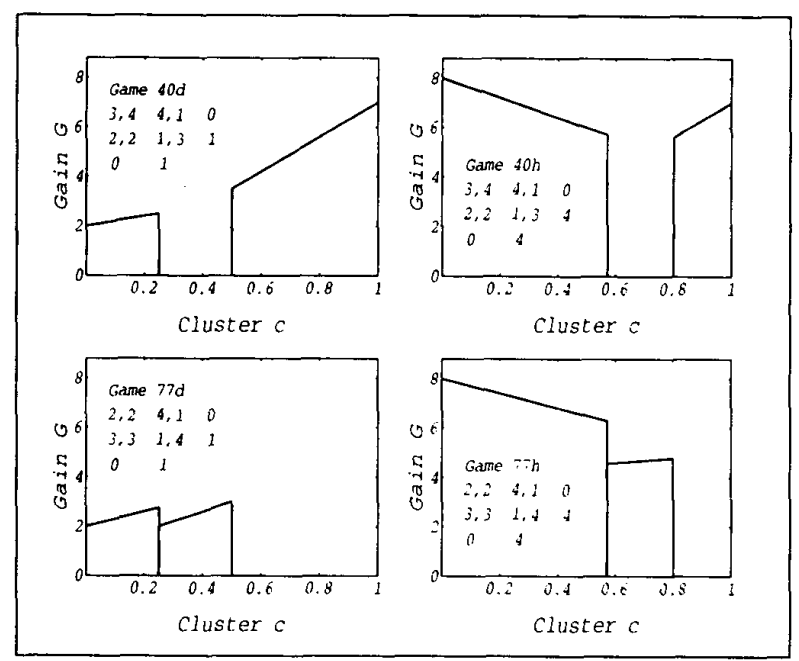

Fig. 7: Games with Weakly Stable Equilibria

$$
\overline{D^{21}}=D^{21} \cdot c+\left[\begin{array}{l}
\left(D^{2}\right)^{T} \\
\left(D^{2}\right)^{T}
\end{array}\right](1-c) .
$$

The gain and its derivative at action pair $\left(\alpha_{1}, \alpha_{2}\right)$ are:

$$
\begin{aligned}
G\left(\alpha_{1}, \alpha_{2}, c\right) & =\overline{D_{\alpha_{1} \alpha_{2}}^{12}}+\overline{D_{\alpha_{1} \alpha_{2}}^{21}} . \\
\frac{d\left(r\left(\alpha_{1}, \alpha_{2}, c\right)\right.}{d c} & =D_{\alpha_{1} \alpha_{2}}^{1 \cdot 2}-D_{\alpha_{1}}^{1}+D_{\alpha_{1} \alpha_{2}}^{21}-D_{\alpha_{2}}^{2} .
\end{aligned}
$$

Since $\frac{d c_{i}\left(\alpha_{1}, \gamma_{2}, c\right)}{d r}$ is a constant, the optimized payoff occurs at $c=1$ or $c=0$ if the derivative is positive or negative, respectively. For $c=1$, the gain is the original 2-player gain, $D_{\left(\gamma_{1} \alpha_{2}\right.}^{12}+D_{\alpha_{1} \alpha_{2}}^{21}$. For $c=0$, the gain is the singleton gains, $D_{\left(r_{1}\right.}^{1}+I_{r_{2}}^{2}$.

Ilowever, by definition, the action pair $\left(\alpha_{1}, \alpha_{2}\right)$ is in equilibrimm if, and only if,

$$
\begin{aligned}
& \left.D_{\alpha_{1} \alpha_{2}}^{12} \cdot r+D\right)_{\alpha_{1}}^{1}(1-c) \geq D_{1-\alpha_{1}, n_{2}}^{12}+D_{1-\alpha_{1}}^{1}(1-c) \\
& D_{r_{1} r_{2}}^{21} \cdot\left(+I_{r_{2}}^{2} \cdot(1-c) \geq D_{\left(r_{1}, 1-r_{2}\right.}^{2 ! 1}+I_{1-n_{2}}^{2}(1-c)\right. \text {. }
\end{aligned}
$$

Solving for $r$ :

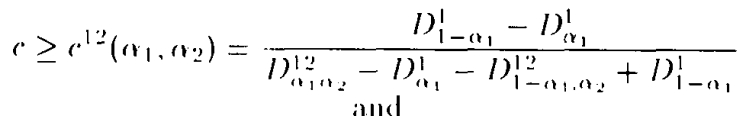

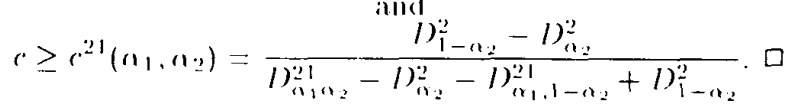

'The theorem states that the gain for an action pair is optimized at $r=1$ or $r=0$ depending on the slope of the gain as a function of e. However, the action pair may not represent an equilibrimu at this value of $c$. We define the system gaim as the gain of an action pair independent of its equilibrium stat.us. 'The cquilibriam gain

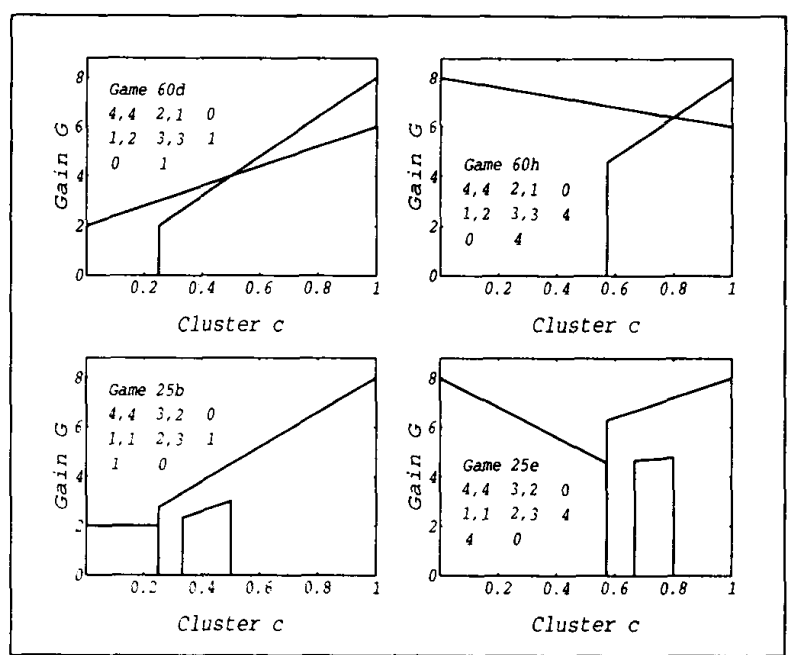

Fig. 8: Games with Concurrent Equilibria

adds the requirement that the action pair represents an equilibrium of the game. Fig. 9 shows system gain and equilibrium gain for an example game. All four action pairs of the game generate a system gain, two of which extend equilibria gains. The optimal equilibrium gain is less than the optimal system gain. This loss is to be expected given the selfish nature of each player.

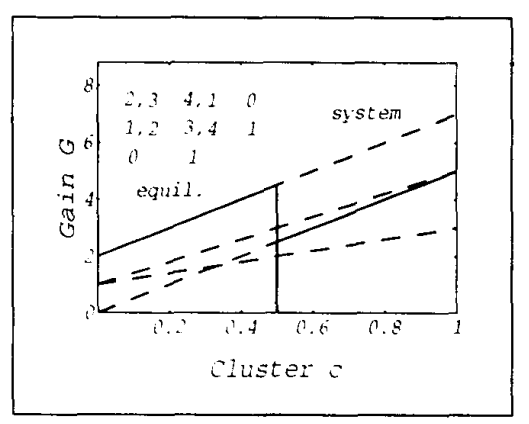

Fig. 9: System Gain and Equilibrium Gain

Fig. 9 illustrates the constraints on equilibria. The action pair $(1,1)$ maintains equilibrium only for $c \leq .5$. 'The action pair $(0,0)$ maintains equilibrium only for $c \geq$ .5. The theorem predicts both of these:

$$
\begin{gathered}
c \geq c^{12}\left(\alpha_{1}, \alpha_{2}\right)=c^{12}(1,1)=\frac{D_{0}^{1}-D_{1}^{1}}{D_{1,1}^{12}-D_{1}^{1}-D_{0,1}^{12}+D_{0}^{1}} \\
=\frac{-1}{-2}
\end{gathered}
$$

that is, $c \leq .5$, and 


$$
\begin{aligned}
& c \geq c^{21}(1,1)=-1 \\
& \quad \text { and } \\
& c \geq c^{12}(0,0)=\frac{1}{2} \\
& \quad \text { and } \\
& c \geq c^{21}(0,0)=\frac{1}{3}
\end{aligned}
$$

The tightest bound on the $(1,1)$ equilibrium is $\leq .5$ (note the change of inequality due to the negative numerator and denominator) and the tightest bound on the $(0,0)$ equilibrium is $c \geq .5$.

\section{Taxonomy of Coalition Games}

Two-player games have been normalized into 78 standard games [9] according to stability: no conflict, strongly stable, strongly stable deficient, stable, threatvulnerable, force-vulnerable, unstable, multiple and none. We now examine $78 \times 4=312$ standard games when one-player games are combined according to our model of probabilistic coalitions and apply our own stability categories of strongly stable, stable and weakly stable. In our normalization, preference values $\{1,2,3,4\}$ are used to designate payoffs in the 2-player game. The values $\{0, \max \}$ are used in the singleton games. A choice of $\max =1$ suggests that the players can perform at the worst case level of the 2-player game. It is also the incremental difference in the 2-player game. The slopes for $\max =1$ are all non-negative implying that the equilibria are induced by the standard $2 \times 2$ game. A choice of $\max =4$ suggests that the players can perform at the best case level of the 2-player game. Figs. 5 to 8 illustrate six of the standard $2 \times 2$ games from [9], each with singletons included for $\max =1$ and $\max =4$. The number of each game corresponds to the original labeling by [9]. Regardless of the choice of $\max =1$ or $\max =4$, both yield the same categorization of each of the 312 games. Although slopes and intersection points may differ, each plot still falls into the same stability, number of equilibria, and concurrency category suggesting a robust set of heuristics. The 312 normalized probabilistic coalition games are categorized by stability, number of equilibria, and concurrency in Table I.

\section{CONCLUSIONS}

Probabilistic coalitions are a simple model for examining the nature of group formation. The probabilistic approach mimics nature and allows the computation of an average game. All games may be classified into one of three categories, each with an implication for agents which make decisions in the context of dynamic group formation. First, strongly stable equilibria games do not present any obstacles for the decision-makers; one
TABLE I: Taxonomy of Standard Coalition Games

\begin{tabular}{cccc} 
\# equilibria & strongly & stable & weakly \\
\hline 1 & $57(18 \%)$ & & $22(7 \%)$ \\
2 & $24(8 \%)$ & $134(43 \%)$ & $19(6 \%)$ \\
3 & & $51(16 \%)$ & $2(1 \%)$ \\
4 & & $3(1 \%)$ & \\
\hline total: 312 & $81(26 \%)$ & $288(60 \%)$ & $43(14 \%)$ \\
\hline \hline concurrent & $24(8 \%)$ & $26(8 \%)$ &
\end{tabular}

set of actions is appropriate regardless of group formation. Second, stable equilibria games present the obstacle that the best action decisions change as the likelihood of group formation changes, but, at least, a pure equilibrium is available to the agents. And last, weakly stable games present the greatest obstacle in that a pure equilibrium may not be available to the agents depending upon the likelihood of group formation.

The uncertainty in group formation may be caused by a variety of factors; in particular, we are interested in examining systems where the adaptive strategies of agents create a probability of group formation and, thus, the agents face the landscape of potential performance illustrated in this study.

\section{RefERENCES}

[1] R. Axelrod and W. Hamilton, "The evolution of cooperation," Science, vol. 211, pp. 1390-1396, Mar. 1981.

[2] B. Hubermann and T. Hogg, "The behavior of computational ecologies," in The Ecology of Computation (B. Hubermann, ed.), North-Holland: Elsevier Science Publishers, 1988.

[3] K. Narendra and M. Thathachar, Learning Automata: An Introduction. Englewood Cliffs, NJ: Prentice-Hall, 1989.

[4] E. Billard and J. Pasquale, "Effects of periodic communication on distributed decision-making," in Proc. 1992 IEEE Int. Conf. Syst, Man, Cybern., pp. 49-54, Oct. 1992

[5] E. Billard, Delayed Communication and Dynamic Group Formation in Distributed Systems. Ph D thesis, University of California, San Diego, 1992

[6] C. Papadimitriou, "On players with a boundesl number of states," Games and Economic Behavior, vol. 4, pp. 122-131, 1992 .

[7] R. Reichardt, "Three-person games with imperfect coalitions: A sociologically relevant concept in game theory," General Systems, vol. 13, pp. 189204,1968

[8] A. Rapoport, N-Person Giame Theory. Ann Arbor, MI: University of Michigan Press, 1970.

[9] A. Rapoport and M. Guyer, " $\Lambda$ taxonomy of $2 \times 2$ games," General Systems: Ycarbook of the Socicty for General Systems Rescarch, vol. 13, pp. 197-210, 1966. 